\title{
Simulation and Experimental Research on a Gas Liquid Separator with Rotary Impeller
}

\author{
Baihui Wang ${ }^{1,2}$, Yingbin $\mathrm{Li}^{2, a}$, Mingjun Deng ${ }^{2}$, Baolu Shi ${ }^{2}$, Wenjin Shang ${ }^{2}$, and Bo $\mathrm{Xu}^{2}$ \\ ${ }^{1}$ College of Aerospace Science and Engineering, National University of Defense Technology, Changsha 410005, China \\ ${ }^{2}$ China Astronaut Research and Training Center, Beijing 100094, China
}

\begin{abstract}
Gas-liquid separation technology under microgravity is the basis for various gas and liquid treatments on a manned spacecraft, which has a wide range of applications in Environmental Control and Life Support System. Dynamic gas-liquid separator is commonly used for the separation of gas-liquid two-phase flow, which has two essential performance parameters called liquid outlet pressure and separating efficiency. Predicting these two parameters accurately under a specific structure has guiding significance for design and application of the dynamic gas-liquid separator. In this study, CFD simulations were conducted using the Volume of Fluid (VOF) model at steady state conditions. In addition, experiments were designed to verify the accuracy of numerical results. Finally, the performance of the separator under microgravity was predicted. It is showed that the simulation method can be utilized to determine the transport performance of dynamic gas-liquid separator, which has significant value in design and optimization.
\end{abstract}

\section{Introduction}

Environmental Control and Life Support System (ELCSS) is the key technology to ensure that astronauts live and work in space environment. Gas-liquid separation is widely used in ECLSS, such as the recovery and treatment of sanitary wastewater, urine and condensate water. Gas-liquid separation technology under microgravity is divided into static gas-liquid separator and dynamic gas-liquid separator according to whether there are moving parts. Compared with static separation, the advantage of dynamic separation is that it can also provide partial pipeline circulation pressure after separation. There is centrifugal motion inside the dynamic separator which is generated by the rotation of gas-liquid mixture in a certain way. Separation will be achieved by centrifugal acceleration and density difference between gas and liquid. It is a key technology to separate gas-liquid mixture by rotary impeller.

The first generation of Mostly Liquid Separator was developed by USA in 1995, which is the application of dynamic separator with rotary impeller for the first time ${ }^{[1]}$. Samplatsky and Dean ${ }^{[2]}$ developed Rotary Separator Accumulator for electrolytic oxygen system in 2002. Murdoch et al. ${ }^{[3]}$ developed Rotary Drum Separator with built-in impeller for Sabatier $\mathrm{CO}_{2}$ reduction system.

The designing difficulty of gas-liquid separator with rotary impeller (GLSRI) is how to determine structure parameters of separating chamber and impellers. In this study, CFD Simulation of GLSRI was carried out, and liquid outlet pressure and separating efficiency were investigated. In the next step, accuracy of the simulation was validated by experimental data with the model machine.

\section{Numerical simulations}

The separation and recovery of condensate is one of the applications of GLSRI. The schematic of condensate collection is shown in Fig.1, where No.1 is a suction pump, which is used to transport the mixture of condensate and air upstream to No.2; No.2 is the GLSRI, which is used to separate condensate and air, and air will be discharged directly into the atmosphere; No.3 is a pressure sensor, which is used to detect the pressure at the liquid outlet of No.2; No.4 is a solenoid valve, which is used to control the condensate pressure and upstream flow, and No.5 is a storage tank, which is used to collect the separated condensate.

Technical indexes of GLSRI are presented in Table 1, in which output pressure of the liquid outlet must be no less than $20 \mathrm{kPa}$. Meanwhile, the volume fraction of gas in separated condensate must be at most $1 \%$.

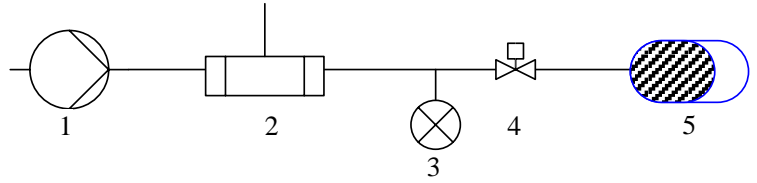

Figure 1. Schematic of condensate water collection.

\footnotetext{
${ }^{\mathrm{a}}$ Corresponding author: liyingbin507@139.com
} 
Table 1. Technical indexes.

\begin{tabular}{|c|c|}
\hline Parameter & Index \\
\hline Components & Mixture of air and condensate \\
\hline Flow & $1 \sim 2 \mathrm{~L} / \mathrm{min}$, about $50 \%$ is air \\
\hline Air volume & Less than $1 \%$ \\
\hline Outlet Pressure & No less than $20 \mathrm{kPa}$ \\
\hline Motor speed & $(1150 \pm 50) \mathrm{rpm}$ \\
\hline
\end{tabular}

\subsection{Geometry modelling and mesh generation}

The fluid domain model was generated from the preliminary design of the structure with a separation chamber and rotary blades according to the technical indexes in Table 1, which is shown in Fig.2(a). Four vertical rotary impellers were adopted, of which the diameter is $140 \mathrm{~mm}$, and the width is $30 \mathrm{~mm}$. Moreover, the chamber can collect $500 \mathrm{ml}$ of water. As shown in Fig.2(b), mesh generation of the fluid domain model was achieved using unstructured grid elements, and the number is 597724 .

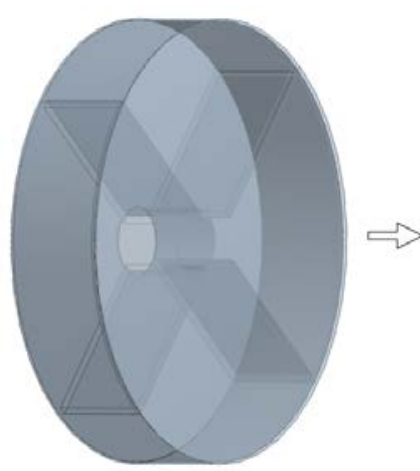

(a) model

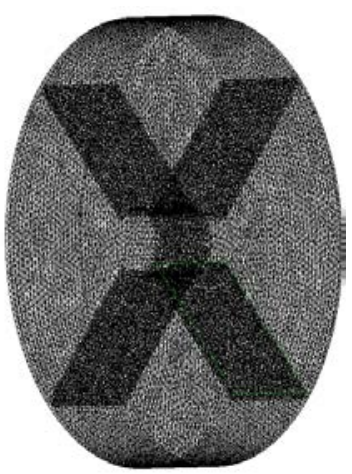

(b) mesh
Figure 2. Fluid domain model and mesh.

\subsection{Numerical method}

\subsubsection{Physical models specification}

VOF model, Mixture model and Euler model are commonly used for multiphase flow analysis. Meanwhile, discrete phase model works when it comes to the calculation of particle flow problems. For the current study, VOF model is selected which can obtain the interface between multiple non-soluble fluids ${ }^{[4]}$.This characteristic can be well used to determine the separation efficiency of GLSRI. In the VOF model, different fluid components share one continuous equation and one momentum, shown in Eq.(1) and Eq.(2).

$$
\frac{\partial \rho}{\partial t}+\nabla \cdot\left(\begin{array}{r}
\mathrm{r} \\
\rho u
\end{array}\right)=S m u
$$

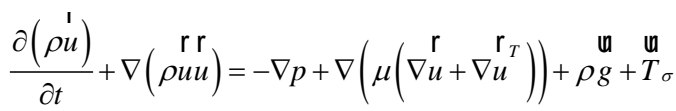

where $\rho$ is the mixture density; $\mu$ is the mixture viscosity; $\dot{u}$ is the mixture velocity; $\stackrel{\mathrm{m}}{T}_{\sigma}$ is the surface tension. The volume fraction occupied by each element of the whole fluid domain.

The surface tension between condensate and air was considered by using the tension model of continuum surface force, and the surface tension coefficient was regarded as $0.073 \mathrm{~N} / \mathrm{m}$. The continuous equation was solved by implicit scheme, which is not limited by courant number and can be solved in steady-state conditions. To prevent diffusion problems caused by low mesh quality, interface Anti-Diffusion should be taken into account. Due to the large body force generated by the rotary impeller, implicit body force is a key factor to improve astringency of the model.

In this study, SST k- $\omega$ turbulence model was used in the simulations, which combines k- $\varepsilon$ model and k- $\omega$ model by weighted average method, using $k-\omega$ model at the wall surface and $k-\varepsilon$ model selecting $k-\omega$ model away from the wall surface. In this way, the dependence of $\omega$ on the relative value of free flow outside the boundary layer in $\mathrm{k}-\omega$ model is overcome ${ }^{[5]}$.

\subsubsection{Boundary conditions and solution methods}

The rotary impeller divides the fluid domain into two parts, that is, the fluid domain that the impeller drives to rotate and the stationary fluid domain at the gap between the rotating fluid domain and the wall. Sliding grid method can be used for the simulation of rotary fluid domain by determining the rotation axis and the speed. The boundary of the rotary fluid domain was modelled as moving wall, of which the relative speed was assumed to be 0 . The remaining wall boundaries were modelled as non-slip walls, and standard wall function method was used to determine the flow near the wall.

In order to determine liquid outlet pressure and separating efficiency, the simulation was carried out in steady-state mode under different working conditions. Coupled algorithm was used for pressure-velocity coupling. Momentum, turbulent kinetic energy and specific dissipation rate were all discrete by using second-order upwind scheme while volume fraction was discrete by using the compressive format.

\section{Simulation results}

When GLSRI is working, the thickness of the liquid increases along the circumference with the continuous injection of condensate until the chamber is filled with condensate. Meanwhile, gas flows out through the gas outlet into the atmosphere. In this study, condensate was replaced by pure water. First, the case that $50 \mathrm{ml}$ of water injected into the chamber was calculated. Then the case of adding another $50 \mathrm{ml}$ of water was recalculated until the chamber was filled with water.

\subsection{Liquid outlet pressure}


The pressure distribution cloud diagrams with different water volume inside the chamber is shown in Fig.3, where the impeller rotates anticlockwise at $1150 \mathrm{rpm}$. It is observed that the pressure near the rotation axis is low, and the pressure near the circumferential boundary is much higher, especially the edge of impeller. The pressure slightly differs between two symmetry points due to the influence of gravity, and the pressure near the gravity is much higher.

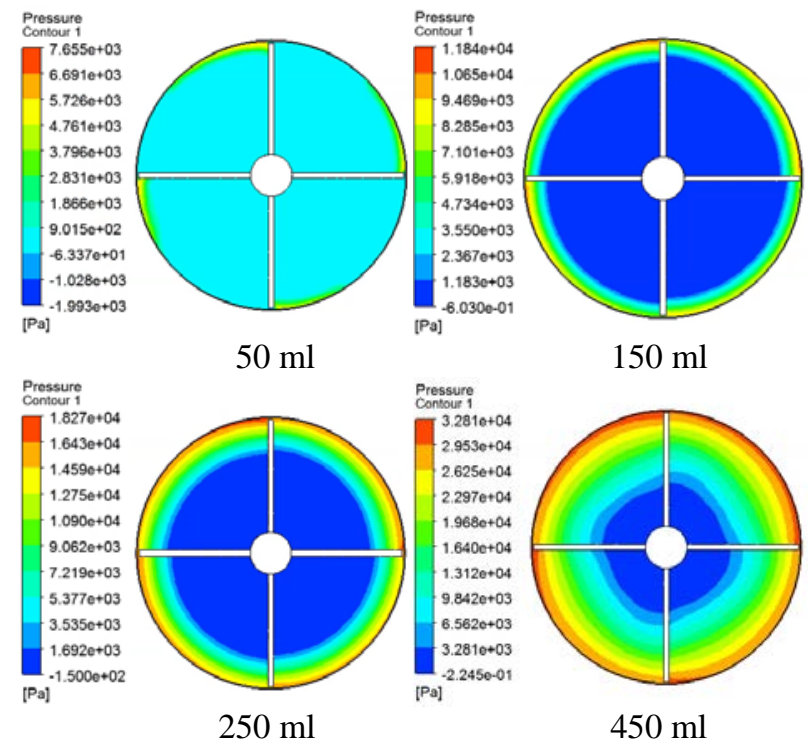

Figure 3. Liquid pressure distribution.

The pressure at the liquid outlet under different water volume is presented in Table 2. It is indicated that the liquid outlet pressure is over $20 \mathrm{kPa}$, which meets the requirement in Table 1 when the water injected reaches $350 \mathrm{ml}$. Therefore, every time GLSRI works, the volume of condensate in the mixture cannot be less than $350 \mathrm{ml}$.

Table 2. Simulation results of liquid outlet pressure.

\begin{tabular}{|c|c|}
\hline Water volume (ml) & Pressure (kPa) \\
\hline 50 & 1.339 \\
\hline 100 & 2.880 \\
\hline 150 & 6.635 \\
\hline 200 & 10.099 \\
\hline 250 & 13.583 \\
\hline 300 & 17.224 \\
\hline 350 & 20.919 \\
\hline 400 & 24.476 \\
\hline 450 & 28.144 \\
\hline 500 & 32.128 \\
\hline
\end{tabular}

\subsection{Separating efficiency}

The separating efficiency of GLSRI is defined as the volume fraction of air in liquid at the liquid outlet. The air volume fraction cloud diagrams with different water injected into the chamber are shown in Fig.4. It is observed that water and air layering is obvious under the effect of the centrifugal force generated by the rotary impeller, indicating that separation of gas-liquid twophase flow can be effectively achieved by this impeller structure. However, under the agitation of the rotary impeller, there is still some air distributed in water. As a result, the gas-liquid mixture cannot be separated completely.

When the water volume in the chamber is $50 \mathrm{ml}$, the liquid outlet is not covered by water completely, so the separating efficiency at this volume was not considered. According to Fig.4, the separating efficiency with different water volume is plotted in Fig.5. It is illustrated that the separating efficiency at each point is lower than $0.6 \%$, which satisfies the requirement of not more than $1 \%$ (shown as the red line in Fig.5) mentioned in Table 1.

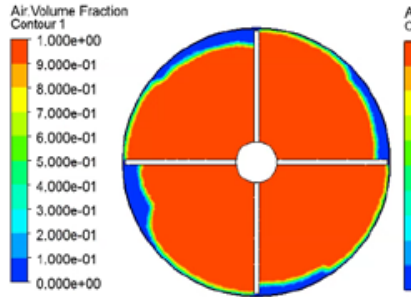

$50 \mathrm{ml}$

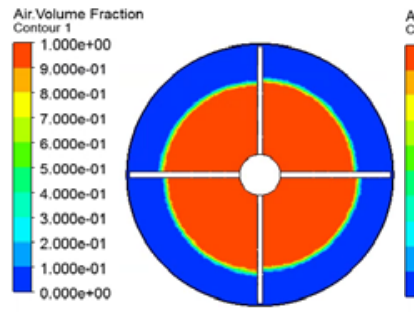

$250 \mathrm{ml}$

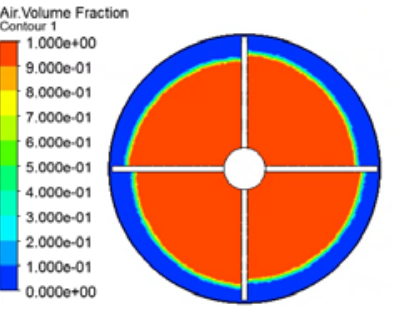

$150 \mathrm{ml}$

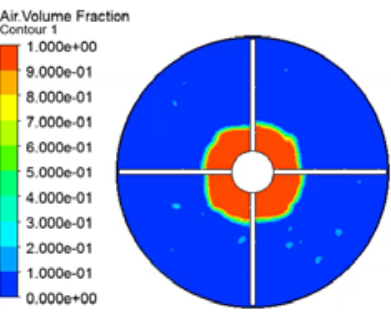

$450 \mathrm{ml}$
Figure 4. Air volume fraction distribution.

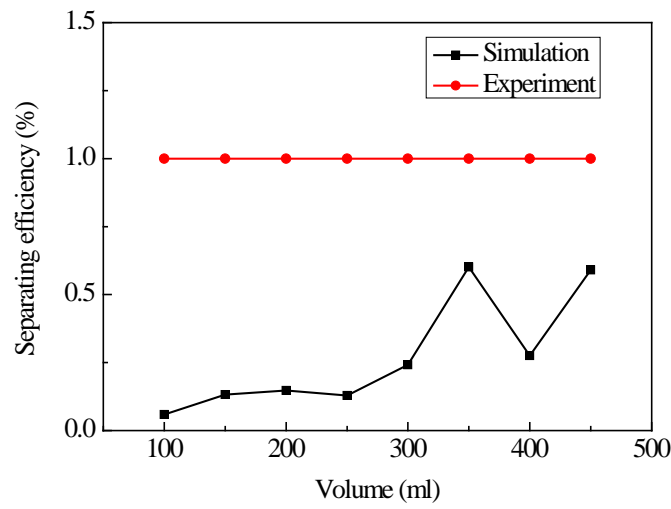

Figure 5. Separating efficiency by simulation.

\section{Experimental verification}

According to the simulation results in Section 3, the preliminary design of GLSRI ${ }^{[6]}$ can meet the technical indexes in Table 1. Therefore, an experimental prototype was designed to verify the accuracy of simulation results. The performance test platform of GLSRI is shown in Fig.6, and the prototype used in experiments is shown in Fig.7. 


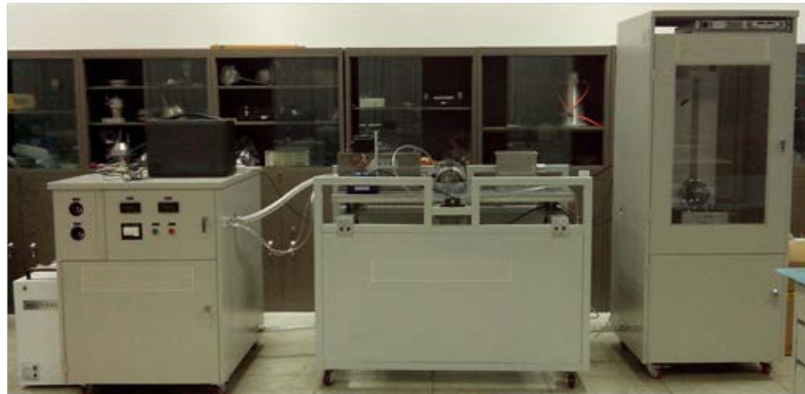

Figure 6. Performance test platform.

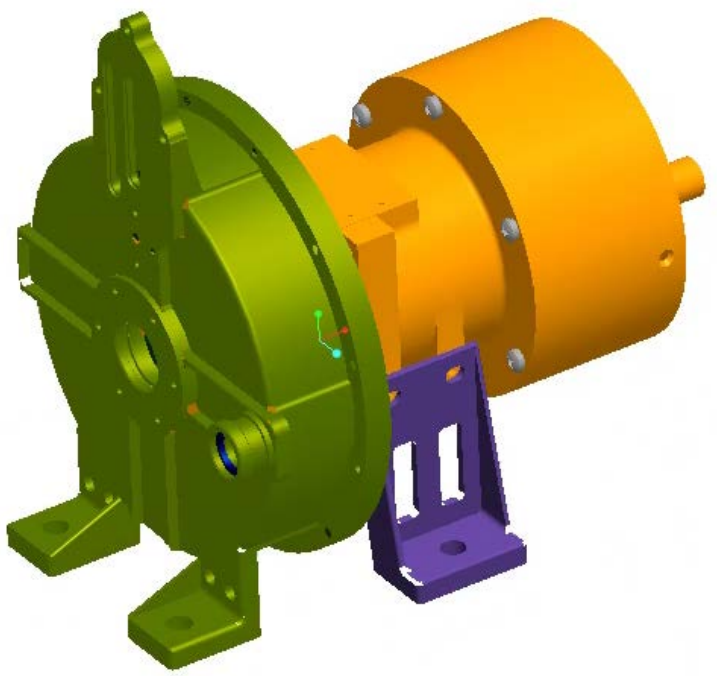

Figure 7. Experimental prototype.

\subsection{Test experiment of liquid outlet pressure}

$50 \mathrm{ml}$ of pure water was injected into separating chamber. Record the value of pressure sensor after the system runs steadily. Increase by $50 \mathrm{ml}$ per time until the volume of water in the chamber reaches $500 \mathrm{ml}$, during which the gas outlet is always open. The liquid outlet pressure values under these 10 water storage states are listed in Table 3.

Table 3. Experimental data of liquid outlet pressure.

\begin{tabular}{|c|c|}
\hline Water volume (ml) & Pressure (kPa) \\
\hline 50 & 0.59 \\
\hline 100 & 3.04 \\
\hline 150 & 6.88 \\
\hline 200 & 10.08 \\
\hline 250 & 13.76 \\
\hline 300 & 17.28 \\
\hline 350 & 20.96 \\
\hline 400 & 24.48 \\
\hline 450 & 28.32 \\
\hline 500 & 31.52 \\
\hline
\end{tabular}

Experimental data in Table 3 are compared with simulation results in Table 2 as shown in Fig.8. It is observed that the pressure of liquid outlet increases gradually with the increase of water volume, which can be regarded as a linear increase. When the water volume is $50 \mathrm{ml}$, the deviation between simulation results and experimental data is large. This is because water volume is not enough to cover the liquid outlet completely, which results in a large fluctuation in the reading of pressure sensor. The error of other points is plotted in Fig.9, in which it can be seen that the maximum error is still less than $6 \%$ and the minimum error is only $0.014 \%$. Moreover, the rising tendency of liquid outlet pressure is the same between simulation results and experimental data. Therefore, it can be illustrated that the CFD simulation of GLSRI in this paper is real and effective.

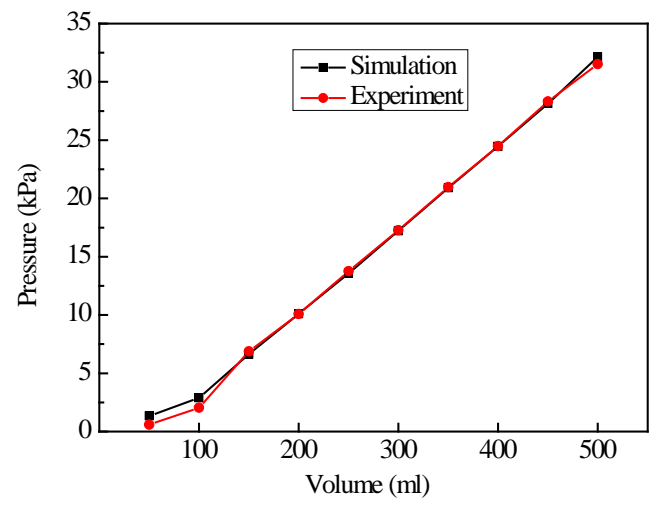

Figure 8. Comparison of liquid outlet pressure.

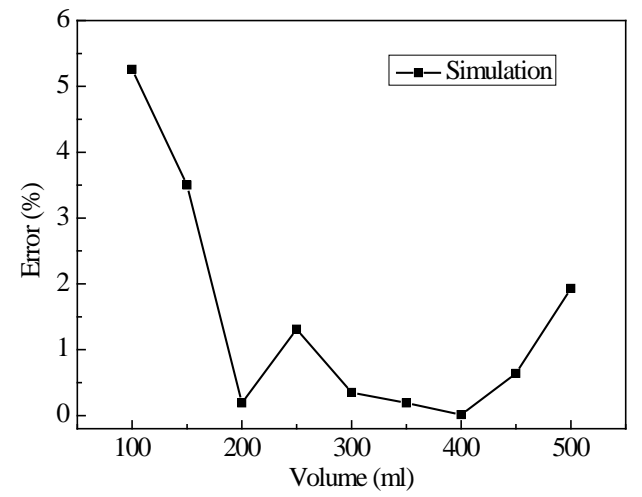

Figure 9. The error of each point.

According to the simulation, the performance of GLSRI under microgravity can be determined, and the relationship between the liquid outlet pressure and water volume was carried out as shown in Fig.10, in which the pressure of liquid outlet was almost the same as that under gravity. 


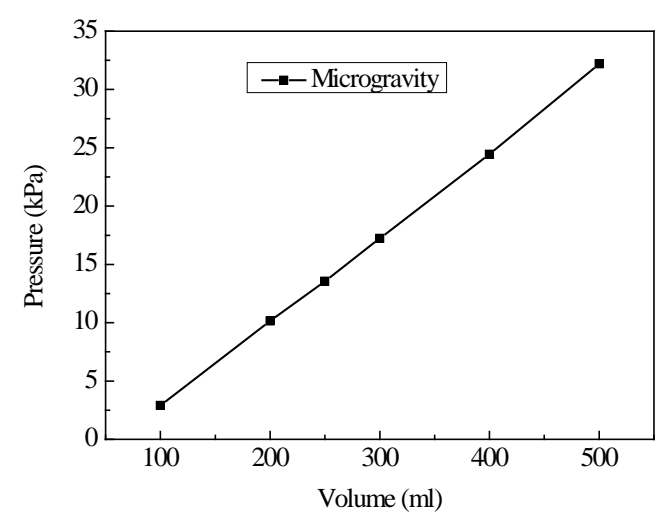

Figure 10. Liquid outlet pressure under microgravity.

\subsection{Test experiment of separating efficiency}

The important components of experimental apparatus are a prototype, a pressure sensor and a measuring cylinder. The schematic of separating efficiency test experiment is shown in Fig.11, where No.1 is a cup for supplying water, whose flow is $2 \mathrm{~L} / \mathrm{min}$; No.2 is a gas-liquid mixer for producing gas-liquid mixture; No.3 is a prototype for the separation of water and air; No.4 is a pressure sensor used for detecting the liquid outlet pressure; No.5 is an electromagnetic valve, the switch of which is controlled by No.5; No.6 is a measuring cylinder, which is used to measure the volume of bubbles in water flowing from the liquid outlet; and No.7 is a power supply.

In order to meet the requirement of separating efficiency, it is necessary to ensure that the liquid outlet is covered by water when opened and closed. According to the liquid pressure curve in Fig.8, during the experiment, when liquid outlet pressure reaches $15 \mathrm{kPa}$, No.5 is open for separation, and when the pressure drops to $7.5 \mathrm{kPa}$, No.5 is closed. Repeat the experiment for several times. Read the bubble volume collected in No.6 to get the separating efficiency. The final average separating efficiency is $0.04 \%$, which meets the requirement that the separating efficiency must be less than $1 \%$, and the accuracy of the CFD simulation is further proved by comparison with Fig.5.

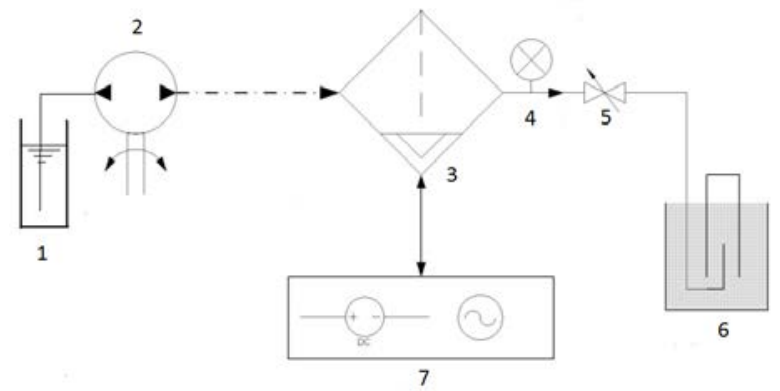

Figure 11. Schematic of separating efficiency test.

\section{Conclusions}

In this paper, a GLSRI that can be used for the separation and recovery of condensate in a manned spacecraft was designed. The internal gas-liquid two-phase flow separation was simulated under steady state conditions combined with the VOF multiphase flow model and sliding grid technology.

The relationship between the liquid outlet pressure and the water volume inside the chamber was obtained. The pressure of liquid outlet increases linearly with the increase of water volume. The liquid outlet pressure exceeds $20 \mathrm{kPa}$ when the water volume reaches $350 \mathrm{ml}$.

The relationship between the separating efficiency and the water volume inside the chamber was obtained. As long as the liquid outlet is covered by water completely, the separating efficiency is less than $1 \%$.

The simulation method is proved to be effective comparing the simulated liquid outlet pressure with experimental results whose maximum error is less than $6 \%$. The comparison of separating efficiency can further illustrate that the simulation method can be used for the prediction of the performance when GLSRI works. And then the performance of GLSRI under microgravity was determined.

As a result, the information get on the ground can be used to evaluate the performance of GLSRI in space, which is significant for the design and application of GLSRI in different situations on a manned spacecraft. In the future work, the separating of gas-liquid mixture under transient conditions will be researched on the basis of this simulation and also the influence of different factors on the performance of GLSRI.

\section{Acknowledgements}

This work was supported by China Astronaut Research and Training Center under project SYFD061909.

\section{References}

1. A.W. Lanzarone, W.C. Dean, S.G. Howard. SAE Technical Paper. No.972374 (1997)

2. D.J. Samplatsky, and W.C. Dean. SAE Technical Paper. No. 2002-01-2360 (2002)

3. K. Murdoch, J. Fort, M. Barone, and D. Holder. SAE Technical Paper. No. 2005-01-2863 (2005)

4. P. Hugo, et al. Journal of Petroleum Science and Engineering, 139, 49-61 (2016)

5. S. Derakhshan, F. Riahi, M. Bashiri. SPE Production \& Operations, 33(02), 320-335 (2018)

6. F. Kulor, E.D. Markus, and M.W. Apprey. International Journal of Mechanical Engineering and Robotics Research, 8, 639-645 (2019) 